\title{
Identifying Multiple Configurations of Complex Molecules in Dynamical Processes: Time Resolved Tunneling Spectroscopy and Density Functional Theory Calculation
}

\author{
Q. Liu, Y. Y. Zhang, N. Jiang, H. G. Zhang, L. Gao, S. X. Du, and H.-J. Gao* \\ Institute of Physics, Chinese Academy of Sciences, Post Office Box 603, Beijing 100190, China
}

(Received 26 October 2009; published 22 April 2010)

\begin{abstract}
We report for the first time a new methodology to determine molecular configurations of a large molecular complex in a dynamical process on a metal surface by combining time-resolved tunneling spectroscopy $(I-t)$ and density functional theory calculation (DFT). Two examples, $(t-\mathrm{Bu})_{4}-\mathrm{ZnPc}$ and $\mathrm{FePc}$, representing molecular rotation and lateral diffusion on $\mathrm{Au}(111)$ surfaces, respectively, were applied to demonstrate our method. Through analysis of statistical occupation time for each configuration, the molecular configuration numbers and energy differences between different configurations of these molecular systems could be unambiguously determined. These experimental results are further compared with DFT calculation to determine corresponding molecular configurations. Importantly, through the spatial $I$ - $t$ mapping, valuable insights of molecular surface diffusion paths are obtained.
\end{abstract}

PACS numbers: 68.43.Jk, 68.37.Ef, 73.20.Hb

The interactions between molecules and metal surfaces have been an important topic in both fundamental science and related device applications, including molecular selfassembly [1,2], charge transport [3,4], etc. In order to gain a full understanding of such fundamental interactions, as a first step, the molecular configuration on a metal surface needs to be accurately determined. To that end, active studies have been carried out for various molecules, but mainly for the static system with small molecules at low temperature $[2,5,6]$. Furthermore, studying molecular configurations in a dynamic process represents more scientific importance but significant technological challenges. Recently, a little progress has been achieved on the dynamical process (including action spectroscopy) of molecular systems [7-15], but mainly for small and simple molecules; however, study on a large molecular complex has been lacking due to insufficient experimental techniques and the intimidating requirement of computation [16]. While the $I-t$ spectroscopy has been successfully applied for investigating motion of single atoms and small molecules $[10,12,13]$, simple extension of such a method to a larger molecule is not straightforward. For example, the size of a large molecule is typically bigger than the tunneling current region confined under the tip apex, which makes it difficult to get all the possible configurations at one site.

In this Letter, we describe a new technique to obtain and analyze $I-t$ spectra with a tip possessing a large apex. Combined with density-functional theory (DFT) calculation, this technique can be applied to identify configurations and corresponding adsorption energies of molecules moving on a surface. Here we use the term "configuration" to represent a specific adsorption state of the molecular system, including the adsorption site on the substrate, molecular conformation and spatial orientation, possible deformation, and so on. With that a full picture of large molecules in a dynamic process can thus be accurately obtained. Two large molecules, $(t-\mathrm{Bu})_{4}-\mathrm{ZnPc}$ and $\mathrm{FePc}$, both on a $\mathrm{Au}(111)$ surface, were chosen to represent two important fundamental motions on surface, rotation, and diffusion, respectively, and were applied as examples to demonstrate the uniqueness of our method.

Experiments were performed using an Omicron UHV MBE-LTSTM system with a base pressure below $3.0 \times$ $10^{-10}$ mbar [17], and all the experiment results were acquired at $80 \mathrm{~K}$. The molecules of $(t-\mathrm{Bu})_{4}-\mathrm{ZnPc}$ and $\mathrm{FePc}$ were purchased from Sigma-Aldrich Inc. Molecules were evaporated to an atomically clean $\mathrm{Au}(111)$ surface using the same technique as described in a previous study [18]. STM images were taken in a constant-current mode and the $I-t$ spectra were recorded with the feedback loop open. The tip with a large apex was produced by gently bringing the tip close to the clean Au substrate and further processed with multiple voltage pulses to achieve stability and reproducibility.

We started with $(t-\mathrm{Bu})_{4}-\mathrm{ZnPc}$ on $\mathrm{Au}(111)$ [Fig. 1(a)]. This molecule was found to rotate at $80 \mathrm{~K}$ without lateral diffusion [Fig. 1(b) shows a typical STM image], but maintained fully stationarity at $5 \mathrm{~K}$ [8]. The $I-t$ spectroscopy measurement was performed at one fixed place, and typical tunneling currents were obtained by frequencycounting statistical analysis used for small molecules in a former study [12]. Figure 1(c) shows a typical $I-t$ spectrum acquired by using a tip of large apex, which shows discrete stepwise tunneling current values with time due to changes of molecular configurations. Figure 1(d) shows subsequent frequency-counting analysis, in which four different current values could be unambiguously distinguished. Thus, the variation of tunneling current could be attributed to the contribution from four different molecular configurations (see supplementary information). While the large tip apex typically leads to poor topographic resolution due to tip convolution [as evident in Fig. 1(b)] [8], its advantage in our current study is that different molecular configurations could be collected during the $I-t$ spectroscopy measure- 


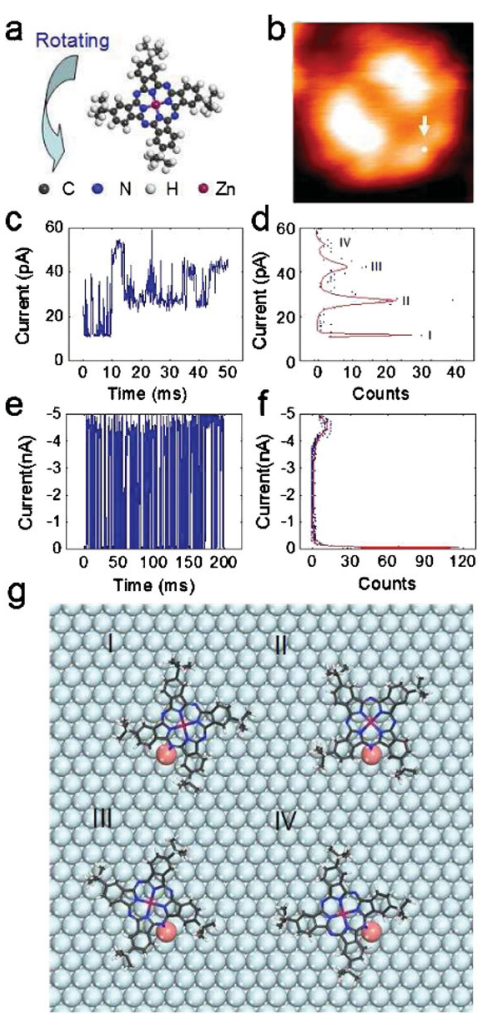

FIG. 1 (color). $\quad \mathrm{A}(t \text { - } \mathrm{Bu})_{4}-\mathrm{ZnPc} / \mathrm{Au}(111)$ system with a rotational motion. (a) Ball and stick model of the $(t-\mathrm{Bu})_{4}-\mathrm{ZnPc}$ molecule. (b) Topography STM image of the $(t-\mathrm{Bu})_{4}-\mathrm{ZnPc}$ molecule on the elbow site of the $\mathrm{Au}(111)$ surface obtained with a blunt tip. Experimental parameters: $3 \mathrm{~nm} \times 3 \mathrm{~nm}, U=$ $-1.67 \mathrm{~V}, I=0.037 \mathrm{nA}$. (c) $I-t$ spectrum measured at the site marked with a bright spot in (b). (d) Frequency-counting statistical analysis of the $I-t$ spectrum according to current values in (c). (e) A typical two level $I-t$ spectrum with a sharp tip. (f) Frequency-counting statistical analysis of the $I-t$ spectrum according to current values in (e). (g) Adsorption configurations provided by the DFT calculations.

ment [Fig. 1(c)]. For comparison, Figs. 1(e) and 1(f) provide results from a control experiment with a sharp tip, in which only two configurations can be resolved in this control experiment, further highlighting the importance as well as advantage of a judicious choice of tip in this study.

The frequency-counting curve was then fit with a set of Lorentz line shapes by approximating the system as a classic system following the Boltzman distribution, and thus the occupation probability of each possible configuration could be derived and summarized in Table I. Importantly, it can be expected that the probability of the current value reflects the stability of molecular configuration. Furthermore, the energy difference between configurations could be derived from these probabilities. For example, according to our measurement, current II in Fig. 1(d) possesses the highest occupation probability. Currents I and III represent the second and the third, respectively. And current IV has the smallest occupation
TABLE I. Comparison of tunneling current, occupation probability, and energy difference between experiments and DFT calculations.

\begin{tabular}{lccccc}
\hline \hline & $i$ state & $I_{i}(\mathrm{nA})$ & $p_{i}$ & $E_{R}^{i}(\mathrm{meV})$ & $E_{\mathrm{CR}}^{i}(\mathrm{meV})$ \\
\hline$(t-\mathrm{Bu})_{4}-\mathrm{ZnPc} / \mathrm{Au}(111)$ & I & 0.011 & $32.6 \%$ & 2 & 9 \\
& II & 0.027 & $44.2 \%$ & 0 & 0 \\
& III & 0.042 & $17.9 \%$ & 6 & 10 \\
$\mathrm{FePc} / \mathrm{Au}(111)$ & IV & 0.053 & $5.3 \%$ & 16 & 12 \\
& I & 0.032 & $7.3 \%$ & 11 & 21 \\
& II & 0.069 & $37.0 \%$ & 0 & 0 \\
& III & 0.092 & $32.1 \%$ & 1 & 10 \\
& IV & 0.124 & $23.6 \%$ & 3.1 & 14 \\
\hline \hline
\end{tabular}

probability. Therefore, we could conclude that the corresponding configuration II is the most stable molecular configuration and thus possesses the lowest adsorption energy on the surface during rotation. The energy difference between configuration IV and configuration II is about $16 \mathrm{meV}$, which is derived from the probabilities (see supplementary information). A similar analysis could also be applied to other configurations and the related energies $\left(E_{R}\right)$ were summarized in Table I. By using this method, we could experimentally obtain the number of configurations and their relative energy difference. An alternative possibility for the observed four current levels is that they were contributed from different spatial regions of a rotating molecule with one configuration. However, if this is correct, the four energies and their corresponding occupation probabilities should be the same and we should not observe statistically a difference, which is different from our experimental observation: Fig. 1(d) shows that the heights, widths, and underlying areas of the four current levels are different from each other, suggesting different occupation probabilities. Therefore, it can be safely concluded that the four current levels come from four different configurations.

First-principle calculations based on DFT were further carried out to examine the experimental results above. In order to achieve more precise results to compare with experiments, we increased the energy cutoff to $500 \mathrm{eV}$ as well as making the grid in real space $30 \%$ denser [8]. The DFT calculation [Fig. 1(g)] also showed the existence of four different configurations on the $\mathrm{Au}(111)$ surface. Importantly, we could directly compare calculated relative energy $\left(E_{\mathrm{CR}}\right)$ with our measurements (Table I), which qualitatively agree with each other [19]. And calculated configurations therefore could be assigned to match with experimental results from Fig. 1(d).

While multiple configurations of the $(t-\mathrm{Bu})_{4}-\mathrm{ZnPc} /$ $\mathrm{Au}(111)$ system could be identified in a rotational process, a more complicated (but more important and general as well) situation is the molecule possessing both diffusion and rotation on a metal surface. We thus extended our method to investigate the FePc molecule [Fig. 2(a)] on the $\mathrm{Au}(111)$ system. Different from the $(t-\mathrm{Bu})_{4}-\mathrm{ZnPc} /$ $\mathrm{Au}(111)$ system, FePc showed dramatically different behavior at $80 \mathrm{~K}$ with small coverage, and molecules could 
not be distinguished in the STM image over a wide range of bias voltage due to the diffusive motion of molecules on the surface [Fig. 2(b)]. Such diffusive behavior made it even more challenging for studying this kind of system [20]. In addition, it should be pointed out that the effect of the bias-induced configuration change should be considered in I- $t$ spectroscopy measurement. In the $(t-\mathrm{Bu})_{4}-\mathrm{ZnPc} / \mathrm{Au}(111)$ system, we tested multiple different bias voltages (positive and negative) and found that generally the voltages did not change the $I-t$ spectrum statistics. For the $\mathrm{FePc} / \mathrm{Au}(111)$ system, we found that higher bias did influence STM images, therefore we chose a small voltage of $0.1 \mathrm{~V}$ to conduct the $I-t$ spectroscopy measurement to reduce such as possible bias effect on the $I-t$ spectra (see supplementary information).

A typical $I-t$ spectrum was shown in the Fig. 2(c), which was acquired at the position marked with a blue spot in Fig. 2(b). Frequency-counting analysis of Fig. 2(c) showed that four typical tunneling currents could be revealed in Fig. 2(d), marked as I, II, III and IV, respectively. While we believed that observed current levels indeed originated from different molecular configurations, another possibility that different currents were contributed from different portions of diffusing molecules in one configuration can be ruled out by the STM image of the FePc molecule with a

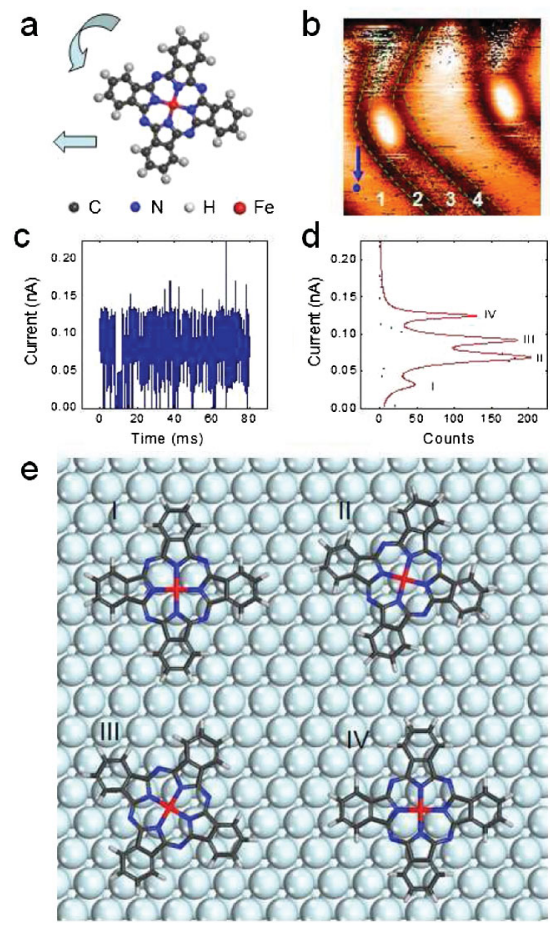

FIG. 2 (color). An FePc/ $\mathrm{Au}(111)$ system with both rotation and diffusion motions. (a) Ball and stick model of a FePc molecule. (b) Topography STM image of $\mathrm{FePc} / \mathrm{Au}(111)$. Experimental parameters: $14 \mathrm{~nm} \times 14 \mathrm{~nm}, U=0.1 \mathrm{~V}, I=$ $0.1 \mathrm{nA}$. (c) $I-t$ spectrum measured at the site marked with a blue spot in (b). (d) Frequency-counting statistical analysis of the $I$ - $t$ spectrum according to current values in (c). (e) Adsorption configurations provided by the DFT calculations. blunt tip (see the last part of the supplementary information). Therefore, we summarized the probabilities of these configurations in the Table I. The four stable adsorption configurations were identified and sorted in an energy sequence compared with those sorted by $E_{R}$ from the $I-t$ spectrum. Calculations based on DFT were also performed for configurations of the FePc molecule on $\mathrm{Au}(111) \mathrm{FCC}$ terrace, and bias voltage factors were not considered in the calculation. $E_{\mathrm{CR}}$ for each configuration was also listed in Table I for comparison [19]. Calculation results were consistent with our previous calculations [17], but with much higher energy precision. Based on the obtained results we can conclude that the most stable configuration in this diffusive system is the configuration II in Fig. 2(e).

We have demonstrated that valuable information of molecular configurations of a large molecular complex on a metal surface can be achieved with our new method. However, another important but more challenging issue is to understand and even predict the molecular diffusion path on the surface. It is worthy of noting that all the results in Figs. 1 and 2 were obtained at a fixed position by recording and analyzing $I-t$ spectra; therefore, in principle, similar recording and analysis could also be performed point-bypoint during the imaging, and thus provide a perspective view of the overall dynamic process of molecular motion on the surface. Our first attempt was demonstrated in Fig. 3 by performing $I-t$ spectroscopy at every pixel in Fig. 2(b) with a total data set of $1024(32 \times 32$ grid in real space $)$. In order to analyze such a large amount of data, a sorted curve analysis method was applied (detailed descriptions and examples are provided in the supplementary information). Briefly, this sorted curve analysis was to sort $I$ - $t$ spectrum based on the logarithm of current values, which could help to overcome the incomparability problem caused by different tip-sample distance at each grid, and could also directly give the current distribution without using frequencycounting method which could be very complicate and time consuming. Typical sorted $I-t$ curves from four different regions were demonstrated in the Fig. 3(a).

With the analysis of sorted curves, energy differences among various molecular configurations could be experimentally determined over the whole surface. Figures 3(b) and $3(\mathrm{~d})$ provide results highlighting $E_{1}$ and $E_{2}$, respectively, $\left(E_{1}\right.$ : the energy difference between the most stable and second stable configurations; $E_{2}$ : the energy difference between the most stable configuration and the third stable configuration). Since the Figs. 3(b) and 3(d) were obtained simultaneously with the topographic image, such energy mapping between different configurations could offer valuable insight on the molecular diffusion process. In order to illustrate this more clearly we showed a 3D presentation of Fig. 3(c). Compared with topographic images, blue regions in the energy mapping mainly corresponded to the FCC terrace of $\mathrm{Au}(111)$ surface [region 1 in Fig. 2(b)]. This suggested that in such area, even though the typical tunneling currents could be quite different due to imaging condition, they showed nearly the same occupation prob- 
a

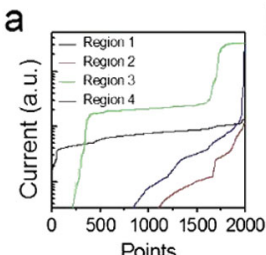

C

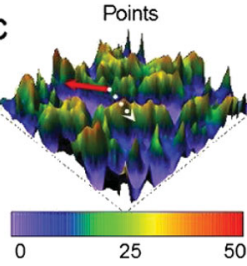

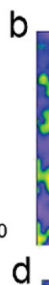
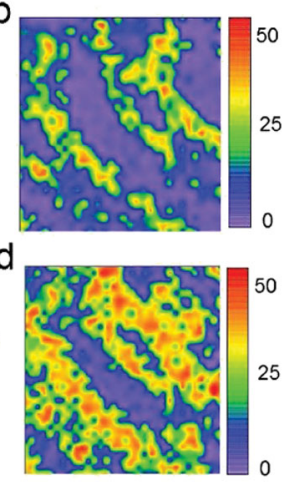

FIG. 3 (color). Mapping of a $\mathrm{FePc} / \mathrm{Au}(111)$ system. (a) Typical sorting curve obtained in different region of $\mathrm{Au}(111)$ herringbone structure. (b) Energy difference between the most stable and second stable configuration in each site in a 2D presentation (graph interpolated). (c) Energy difference between the most stable and second stable configuration in each site in a 3D presentation. (d) Energy difference between the most stable and third stable configuration in each site in a $2 \mathrm{D}$ presentation (graph interpolated). Color bar is in the unit of one $\mathrm{meV}$.

abilities and diffusion behavior with a relative low $E_{1}$ and $E_{2}$ (see the supplementary information). This further implied that FePc molecule could diffuse easily in this region by toggling between most and second stable configurations (as highlighted by the red solid arrow) (even though the third configuration might be taken during the diffusion but with lower probability). On the other hand, in the boundary region between FCC and HCP on the Au(111) surface, both $E_{1}$ and $E_{2}$ were large, which could be evident from the color scheme, suggesting that in this area the adsorption structures at a certain region had a large energy difference and the FePc molecule had a low possibility to cross the domain wall [yellow dashed arrow in Fig. 3(c)]. By using such method, surface diffusion barrier could be extracted experimentally, and this would offer a real picture of surface diffusion process of molecule when further combined with DFT calculations. Compared with traditional direct DFT-based diffusion calculation and MM/MD based calculation [16,21], our method could significantly reduce the computation time and provide more insightful results.

In summary, we have demonstrated a new methodology by combining $I-t$ spectroscopy and DFT calculations for studying different motions of large complex molecules on metal surfaces. For the first time we are able to identify different molecular configurations with different occupation probabilities in a dynamic process of a large molecular complex. Determination of molecular configurations represents the first step toward full understanding of different phenomena (such as diffusion, reaction, catalysis, etc.) on the surface and various interactions between molecules and metal surfaces. Importantly, combined with mapping tech-

niques, our method could be further extended for understanding the diffusion path of molecules on the surface, which has been a challenging problem due to the lack of efficient experimental techniques as well as the demanding need of computation. Furthermore, while the current study focused on the energy difference among multiple configurations, it has been demonstrated that accurate energy barriers can be potentially determined by analyzing timebin counting in the $I-t$ spectra [22]. If such analysis can be simultaneously integrated with our current method, a complete description of the molecular dynamics of a large molecular complex should be immediately accessible.

We are grateful to W. Ho for helpful discussion. Work at IOP was supported by the NSFC and "973" Projects of China, CAS, and Shanghai Supercomputer Center.

*hjgao@iphy.ac.cn

[1] J. V. Barth, G. Costantini, and K. Kern, Nature (London) 437, 671 (2005).

[2] M. Eremtchenko, J. A. Schaefer, and F. S. Tautz, Nature (London) 425, 602 (2003).

[3] G. V. Nazin, S. W. Wu, and W. Ho, Proc. Natl. Acad. Sci. U.S.A. 102, 8832 (2005).

[4] N.P. Guisinger, N. L. Yoder, and M.C. Hersam, Proc. Natl. Acad. Sci. U.S.A. 102, 8838 (2005).

[5] J. Repp et al., Phys. Rev. Lett. 94, 026803 (2005).

[6] N. Ogawa, G. Mikaelian, and W. Ho, Phys. Rev. Lett. 98, 166103 (2007).

[7] J. K. Gimzewski et al., Science 281, 531 (1998).

[8] L. Gao et al., Phys. Rev. Lett. 101, 197209 (2008).

[9] H. Dil et al., Science 319, 1824 (2008).

[10] B. C. Stipe, M. A. Rezaei, and W. Ho, Phys. Rev. Lett. 81, 1263 (1998).

[11] P. Han et al., J. Am. Chem. Soc. 126, 10787 (2004).

[12] J. A. Stroscio and R. J. Celotta, Science 306, 242 (2004).

[13] A. Saedi et al., Nano Lett. 9, 1733 (2009).

[14] S. Katano et al., J. Phys. Chem. C 113, 19277 (2009).

[15] Y. Sainoo et al., Phys. Rev. Lett. 95, 246102 (2005).

[16] G. Henkelman et al., Phys. Rev. B 72, 115437 (2005).

[17] L. Gao et al., Phys. Rev. Lett. 99, 106402 (2007).

[18] Y.L. Wang et al., Phys. Rev. B 69, 075408 (2004).

[19] See supplementary material at http://link.aps.org/ supplemental/10.1103/PhysRevLett.104.166101. There are still noticeable differences, and detailed are discussed here.

[20] For small molecules with slow motion, a related study can be achieved by the tracking technique as in [22] and E. Hill, B. Freelon, and E. Ganz, Phys. Rev. B 60, 15896 (1999). However, the tracking technique requires a special algorithm and ultrafast scanning speed. Especially for such a highly diffusive molecular system, it will be difficult for a tracking method.

[21] P. Fouqueta et al., Carbon 47, 2627 (2009).

[22] W. Ho, J. Chem. Phys. 117, 11033 (2002). 